\title{
Autotransplantation of premolars
}

\author{
G. Mensink ${ }^{1}$ and R. van Merkesteyn ${ }^{2}$
}

- Autotransplantation is easy to perform surgically and the treatment is well tolerated by young patients.

- Autotransplantation is aesthetically superior and more cost-effective than other treatments like fixed prostheses and osseo-integrated implants.

- Success of autotransplantation depends on careful management during the surgical procedure, especially of the periodontal ligament.

\begin{abstract}
Autotransplantation is a reliable treatment option for the replacement of missing teeth; however, there is a wide variation in reported survival rates. The purpose of this study was to evaluate the success and survival rate of premolar autotransplantation and to underline the importance of autotransplantation in the treatment of missing teeth. We present the treatment and follow-up of 63 premolar autotransplants in 44 patients. After transplantation under local anaesthesia, radiological and clinical follow-up showed a survival rate of 100\%, ie all premolars were still functional. We conclude that autotransplantation of premolars is a reliable treatment method especially for agenesis. It is not difficult to perform and is aesthetically superior and more cost-effective than other treatments, especially when orthodontic alignment is necessary.
\end{abstract}

\section{INTRODUCTION}

Common indications for autotransplantation include the transplantation of impacted teeth to their normal position, agenesis of teeth, lost teeth (as a result of caries or periodontal and periapical lesions) and trauma. ${ }^{1,2}$ In children, the most common indication, agenesis usually involves the second premolars. Growth and developmental changes are complicating factors which may pose a challenge to premolar replacement. ${ }^{3,4}$ The potential for orthodontic alignment after autotransplantation of these teeth offers a great advantage.

Autotransplantation is described as an effective treatment for the replacement of missing teeth. Survival rates vary from 74-100\%.4-8 Success depends on careful management, especially of the periodontal ligament, during the surgical procedure. Maintaining an intact periodontal ligament during transplantation aids bone induction, prevents root resorption (replacement and surface resorption) and creates the possibility of orthodontic movement. ${ }^{4,6}$

$1,2^{*}$ Oral and Maxillofacial Surgery, Leiden University Medical Center, Albinusdreef 2, Leiden, 2300 RC, Netherlands

${ }^{*}$ Correspondence to: Professor Richard van Merkesteyn Email: j.p.r.van_merkesteyn@lumc.nl

\section{Refereed Paper}

Accepted 10 December 2009

DOI: $10.1038 /$ sj.bdj.2010.102

${ }^{\circledR}$ British Dental Journal 2010; 208: 109-111
In this report, we present 44 patients with 63 premolar autotransplants. The purpose of this study was to evaluate the success and survival rate of autotransplantation, and to emphasise to dentists, orthodontists and maxillofacial surgeons that this is an important procedure for the treatment of missing teeth.

\section{MATERIALS AND METHODS}

We undertook a retrospective review of surgical records held by the Department of Oral and Maxillofacial Surgery of the Leiden University Medical Centre and identified all patients who had undergone autotransplantation of premolars between 1995 and 2004. A patient was considered eligible for the study if the records were adequate, ie they included the date of transplantation, a description of transplantation methods and follow-up records with radiographic examinations. Only patients with a follow-up duration of at least one year were included in the study.

From the records, we identified 48 patients with a total of 67 transplanted premolars. However, four cases did not meet the follow-up criteria and were excluded. As a result, the final study population consisted of 44 patients with a total of 63 transplanted teeth. Duration of follow-up ranged from 1-5 years with a mean of 1.76 years, and the age of the patients varied from 10-31 years with a mean of 13 years. There were 18 male and 26 female patients. All patients had been referred by an orthodontist or dental practitioner. In all cases, premolars were transplanted to another premolar site.

Pre-operative orthopantomograms (OPTs) or single-tooth radiographs were available for all patients. Whenever possible, surgery was planned when the length of the apex was between a half and three-quarters of its estimated final length. Fifty-three (84.1\%) donor teeth showed a root formation of a half to threequarters at the time of transplantation. Because of late referrals, six teeth were transplanted after root formation was three-quarters completed.

The most common indication (95\%) was agenesis of a lower second premolar in combination with a class II malocclusion. Root curvature and bucco-palatal dimensions determined whether the first or second maxillary premolar was selected for transplantation.

In the majority of cases, the transplantations were performed under local anaesthesia. Antibiotic prophylaxis was administered before surgery in all patients (amoxicillin $500 \mathrm{mg}$ orally, thrice daily for one week).

Using local anaesthesia, the primary tooth on the acceptor site was gently 
removed with forceps. Care was taken to prevent damage to the periodontal ligaments. After limited elevation of the periosteum of the donor site, the tooth was preserved in saline while a neo-alveolus was prepared with a bur. When the deciduous molar was extracted in the same session before transplantation, no periosteal elevation was performed. The alveolus was fashioned until the premolar fitted with slight occlusal pressure just caudally of the occlusal plane. A rotation of 90 or 180 degrees was considered in cases with curved roots or when necessary to achieve a better fit to the surrounding bone. After transplantation, the tooth was fixed with a Vicryl $3 \times 0$ suture (Ethicon/Johnson \& Johnson, Amersfoort, The Netherlands) crossing from the mesial to the distal gingival papilla. Care was taken to prevent interocclusal contact with the newlyplaced tooth.

After surgery, the patient was prescribed chlorhexidine and analgesics, and was provided with instructions on soft diet and oral hygiene. Post-operative followup consisted of radiographs and clinical examinations at one week, six months and 12 months post-surgery (Fig. 1). After one week, the sutures were removed. Clinical examination included assessment of any colour change in the tooth, mobility and position, as well as percussion and probing of the gingiva. The radiographs were evaluated for signs of internal or external root resorption, periapical pathology, root canal obliteration, marginal bone loss and root development (Fig. 1). If internal or external root resorption or periapical disease was present, the patient was referred to a dentist for endodontic treatment.

In the radiographs shown in this paper, agenesis of the 45 is visible (Fig. 1a). The 25 was transplanted. Inadequate root development is present in the second premolar on the right. The alveolus of the 85 is still present (Fig. 1b). After six months no pathology is present, the alveolus of the 85 is filled out with bone, root obliteration becomes apparent and some root development is visible at the apex (Fig. 1c).

\section{RESULTS}

All of the 63 premolars were functional 12 months post-transplant. One tooth had a lingual pocket which was treated. None of the premolars showed colour changes. No pathological mobility was found. In 12 premolars (19\%) a high percussion sound was observed. At the request of the orthodontist, three of these were slightly luxated in a later session because of infraposition.

Three premolars (5\%) needed endodontic treatment to arrest periapical resorption. One tooth was endodontically treated preoperatively because of total root formation at transplantation. Fifty-six premolars (89\%) showed more or less obliteration of the root canal. Thirty-eight premolars $(60 \%)$ showed lengthening of the root after transplantation. Sixty-two transplants (98\%) were orthodontically moved after transplantation.

\section{DISCUSSION}

Autotransplantation of premolars is considered a superior treatment in cases of agenesis of lower second premolars in combination with class II malocclusion. The potential for orthodontic movement of transplanted premolars is a great advantage in children in comparison to other treatment options such as osseointegrated implants. Despite the high success rates of implants nowadays, growth in children will result in movement of the teeth. Eruption or orthodontic alignment is not compatible with rigid implants. Rehabilitation with fixed prostheses (crowns and bridges) is also a possibility, but the extended preparation required for what are usually healthy teeth and the natural lifespan of these restorations (about ten to 20 years) makes them less suitable., ${ }^{5,6}$

For these reasons, patients presenting before the pubertal growth phase should ideally benefit from autotransplantation. However, autotransplantation should not be carried out at too early a stage of root development, because the prognosis is not as predictable.

The reported survival and success rates of transplanted teeth vary from 74-100\%.0.8 Careful removal of the donor tooth with preservation of a viable periodontal ligament is considered crucial. Placement of the transplanted tooth without pressure in a well-formed neo-alveolus is also considered a key to success. ${ }^{2,67}$ These factors were also considered important in our series, resulting in similarly high success rates.

Some researchers describe positioning of

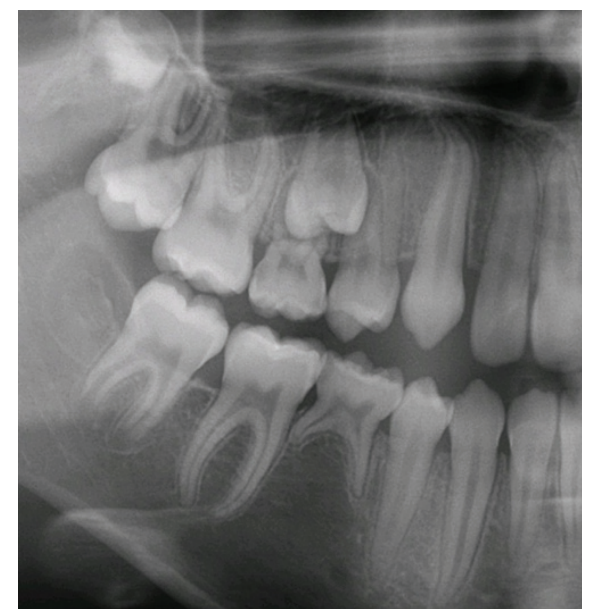

Fig. 1a Pre-operative OPT: agenesis of a 45. For this reason the impacted 25 was autotransplanted to location 45

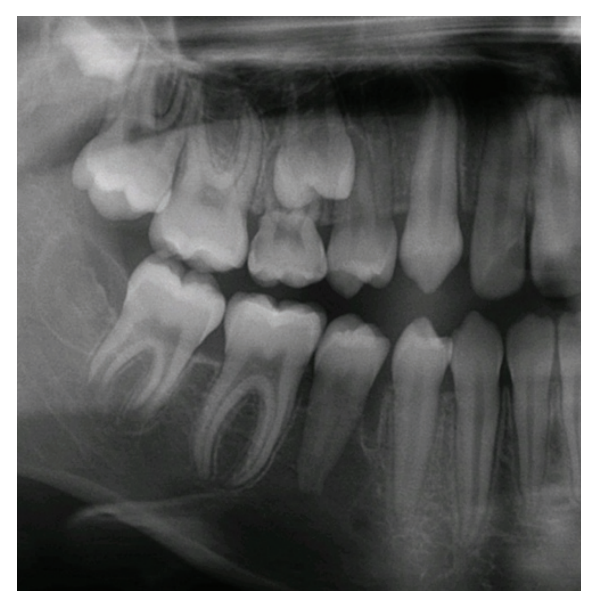

Fig. 16 Post-operative OPT: one week after autotransplantation of the 25 . Note the still visible alveolus of the 85. After the alveolus was prepared, the premolar was placed until it fitted with slight occlusal pressure just caudally of the occlusal plane For further clinical discussion, see Andreasen et $a l_{l^{6}, 7,8,11,15}$ and Tsukiboshi ${ }^{2,6}$

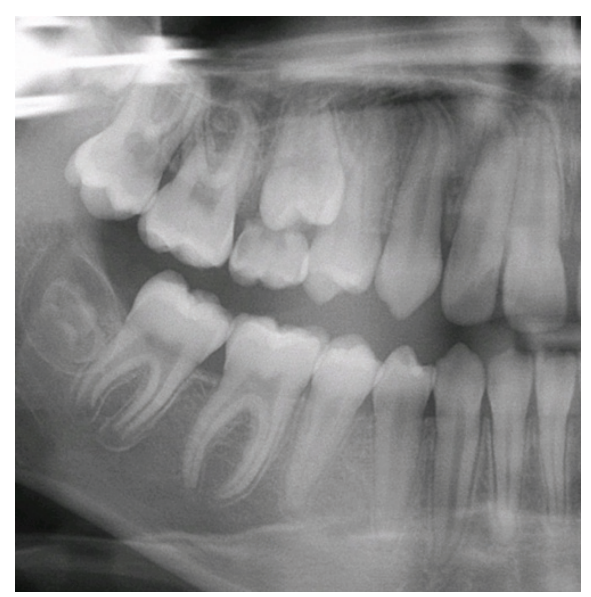

Fig. 1c Post-operative OPT: six months after surgery. Obliteration of the root canal and further root formation can be seen. No internal or external root resorption or periapical pathology are present. Note the bone formation in the previous alveolus of the $\mathbf{8 5}$ 
the transplant back in its original socket during preparation of the neo-alveolus. ${ }^{4,6}$ During our procedures, the transplants were preserved in saline. We conclude from the similar success rates that either storage possibility may be considered. Most researchers advise that surgery should be performed when the root formation measures half to three-quarters of the final root length, to stimulate re-vascularisation of the pulp. .,69-11 $^{1}$ There were very few teeth in our study which did not fulfil this criterion, and the transplants succeeded without any problems.

Root development of the transplanted teeth can be expected if Hertwig's epithelial sheath is preserved around the apices. Sixty percent of the premolars showed an increased root length in our study (Fig. 1). This is in line with the findings of previous studies. ${ }^{6,8,12}$

In this retrospective study, root development was examined on OPTs or single-tooth radiographs taken by different technicians. These differences could influence the interpretation of the root length. In this study, we used suture fixation of the premolars. It has been reported that the ingrowth of new vessels is promoted by small movements of the transplant, and therefore rigid fixation is thought to have a negative influence on the revascularisation process; it has a negative influence on final root length and root length increment. ${ }^{13}$ The finding that 60\% of the premolars in this study showed an increased root length seems to confirm this statement.

Root obliteration is considered to be an indicator of vitality (Fig. 1). Growth of well vascularised connective tissue in the root canal causes formation of tertiary dentine. This tissue is vital and will not induce pathological changes. ${ }^{6,12,15-17}$ Therefore, endodontic treatment was started only when pathological changes were observed in the periapical region and not on obliteration of the pulp canal only. ${ }^{15}$ Three of our patients needed endodontic treatment to arrest periapical inflammation.

It was noted that one premolar developed a lingual pocket that was probably caused by surgical damage to the periodontium. This proved treatable and the premolar was functioning well during follow-up.

Patients were administered antibiotics before the surgery. Because of the high success rate, there may be grounds for omitting this prophylaxis, which would also be in keeping with a current trend away from administering antibiotics in dental implant surgery.

In the reported series of patients, orthodontic treatment was started after three months. Of the transplanted premolars, 98\% were successfully moved orthodontically. This is in line with Paulsen et al., ${ }^{12}$ who advise starting after periodontal healing but before total pulp canal obliteration, ie 3-9 months after transplantation.

A few teeth showed a higher percussion sound (19\%). However, during orthodontic treatment only three premolars were markedly ankylosed and required luxation, after which they were successfully moved.

\section{CONCLUSION}

Autotransplantation of premolars is a reliable treatment for agenesis of premolars. Its potential to accommodate growth and orthodontic treatment is an important factor favouring autotransplantation above other treatment options. Autotransplantation is surgically easy to perform. The treatment is well-tolerated by young patients and is both aesthetically superior and more cost-effective, especially when orthodontic alignment is necessary.

1. Kallu R, Vinckier F, Politis C, Mwalili S, Willems G. Tooth transplantations: a descriptive retrospective study. Int J Oral Maxillofac Surg 2005; 34: 745-755.

2. Tsukiboshi M. Autotransplantation of teeth. Chicago: Quintessence Publishing, 2001.

3. Mattheeuws N, Dermaut L, Martens G. Has hypodontia increased in Caucasians during the 20th century? A meta-analysis. Eur J Orthod 2004; 26: 99-103.

4. Jonssen T, Sigurdsson T J. Autotransplantation of premolars to premolar sites. A long-term follow-up study of 40 consecutive patients. Am J Orthod Dentofacial Orthop 2004; 125: 668-675.

5. Czochrowska E M, Stenvik A. Outcome of tooth transplantation: survival and success rates 17-41 years posttreatment. Am J Orthod Dentofacial Orthop 2002; 121: 110-119.

6. Tsukiboshi M. Autotransplantation of teeth requirements for predictable success. Dent Traumato/ 2002; 18: 157-180.

7. Andreasen J O, Paulsen H U, Yu Z, Bayer T, Schwartz 0 . A long-term study of 370 autotransplanted premolars. Part II. Tooth survival and pulp healing subsequent to transplantation. Eur J Orthod 1990; 12: 14-24.

8. Andreasen J O, Paulsen H U, Yu Z, Bayer T. A longterm study of 370 autotransplanted premolars. Part IV. Root development subsequent to transplantation. Eur J Orthod 1990; 12: 38-50.

9. Schwartz O, Bergmann P, Klausen B. Resorption of autotransplanted human teeth: a retrospective study of 291 transplantations over a period of 25 years. Int Endod J 1985; 18: 119-131.

10. Ploder O, Partik B, Rand T, Fock N et al. Reperfusion of autotransplanted teeth-comparison of clinical measurements by means of dental magnetic resonance imaging. Oral Surg Oral Med Oral Pathol Oral Radiol Endod 2001; 92: 335-340.

11. Andreasen J O, Paulsen H U, Yu Z, Ahlquist R et al. A long-term study of 370 autotransplanted premolars. Part I. Surgical procedures and standardized techniques for monitoring healing. Eur J Orthod 1990; 12: 3-13.

12. Paulsen H U, Andreasen J O, Schwartz O. Pulp and periodontal healing, root development and root resorption subsequent to transplantation and orthodontic rotation: a long-term study of autotransplanted premolars. Am J Orthod Dentofacial Orthop 1995; 108: 630-640.

13. Bauss 0, Swestka-Polly R. Effect of different splinting methods and fixation periods on root development of autotransplanted immature third molars. J Oral Maxillofac Surg 2005 ; 63: 304-310.

14. Waikakul A, Punwutikorn J, Kasetsuwan J. Response of autotransplanted teeth to electric pulp testing. Oral Surg Oral Med Oral Pathol Oral Radiol Endod 2002; 94: 249-255.

15. Andreasen J O, Paulsen H U, Yu Z, Schwartz O. A long-term study of 370 autotransplanted premolars. Part III. Periodontal healing subsequent to transplantation. Eur J Orthod 1990; 12: 25-23.

16. Skoglund A, Hasselgren G. Tissue changes in immature dog teeth autotransplanted to surgically prepared sockets. Oral Surg Oral Med Oral Pathol 1992; 74: 789-795.

17. Skoglund A, Tronstad L, Wallenius K. A micro-angiographic study of vascular changes in replanted and autotransplanted teeth of young dogs. Oral Surg Oral Med Oral Pathol 1978; 45: 17-28. 